\title{
EXPERIENCIA EN LA EVALUACIÓN DE IMPACTO AMBIENTAL DE CARRETERAS
}

\author{
(EXPERIENCE ON ENVIRONMENTAL IMPACT ESSESSMENT \\ OF ROADS)
}

Domingo Gómez Orea, Dr. Ingeniero Agrónomo - U.P.M.

Teresa Villarino Valdivielso, Dr. Ingeniero de Montes - MELISSA, S.A.

ESPAÑA

RESUMEN

El presente articulo pasa revista a los problemas técnicos y administrativos que presenta en España la aplicación del procedimiento de Evaluación del Impacto Ambiental en base a la experiencia acumulada durante los últimos cinco años. Se presta especial atención al caso de las grandes infraestructuras viarias.

\section{SUMMARY}

This article reviews technical and administrative problems to be dealt with in Spain in the application of the procedure of Environmental Impact Assessment based on the experience of the last five years. Special attention is paid to main road infrastructures.

\section{INTRODUCCIÓN}

Este artículo presenta una serie de reflexiones subjetivas fruto de la experiencia directa, en los últimos años, en materia de EIA; el punto de vista desde el que se ha redactado es el del profesional independiente en funciones de consultoría, preocupado porque el poderoso instrumento que es la EIA sea utilizado de la forma más adecuada. Además, incorpora reflexiones extraídas del Libro Verde de la Comisión Europea sobre el impacto del transporte en el medio ambiente, así como recomendaciones y criterios del
V Programa Comunitario de Política de Actuación en Materia de Medio Ambiente y Desarrollo Sostenible.

Se parte de la idea de que, más que la incorporación de un conjunto de variables, la gestión ambiental implica un estilo, una forma de entender y de aproximarse a los problemas y a sus soluciones. Que un proyecto no es concebible si no es contextualizado en un entorno con el que configura un único sistema; proyecto y entorno no son entidades contrapuestas sino aspectos indisociables de tina única realidad. 


\section{Misa contra el verte-
dero. Unos trescientos veci-
nos de cuatro parroquias asis-
tieron ayer a una misa en la
catedral de Lugo y pidieron a la
divinidad que se paralicen las
obras de construcción del verte-
dero controlado de residuos só-
lidos urbanos. El vecindario de
las parroquias de Teixeiro, La-
vio, Benade y Rubias, que llegó
a la capital lucense en autoca-
res, se concentró en la catedral
y asistióa una misa oficiada por
el obispo de la diócesis, fray
José Gómez. D. $\mathbf{M . 1 2 . 8 7}$}

El impacto social : puede leerse carreteras donde dice vertedero.

En contra de la consideración unitaria del proyecto y de su entorno, la legislación en materia de EIA, y la consiguiente gestión/control de este poderoso instrumento, está escorada descaradamente hacia el efecto del proyecto sobre el entorno, y casi con exclusividad a los impactos de signo negativo, excluyendo las relaciones de sentido contrario, es decir, el comportamiento del entorno respecto al proyecto. Ello parece ser así porque:

a. en el caso de los riesgos o amenazas del entorno sobre el proyecto, se entiende que habrán sido contemplados por el proyectista, y

b. en lo que respecta a la adaptación del proyecto a la vocacionalidad del medio, parece no interesar. Ello a pesar de que el espíritu de la legislación propugna una intervención de la EIA en la generación de alternativas. Sin embargo el articulado restringe la intervención de la EIA a la solución elegida por la autoridad sustantiva, sobre la que se hace la Declaración de Impacto Ambiental. Resulta así que la fuerza con que intervenga la EIA en el proceso del proyecto depende en gran medida de tal autoridad sustantiva; consecuencia de ello es la restricción de las funciones de la EIA a la simple corrección de fallos graves, en el mejor de los casos, permaneciendo muy alejada de la intervención en el diseño.

Así aplicada la EIA se convierte en un documento reactivo, antipático para el promotor, para el proyectista y para el órgano sustantivo, que la entienden como una ingerencia molesta en asuntos de su especialidad profesional y de su competencia. Paralelamente el experto ambiental se siente intelectualmente defraudado ante la escasa consideración de su trabajo y la reducción de su aportación al papel de mero trámite.

\section{LA CONTRAPOSICIÓN \\ DE LOS AGENTES DE LA EIA}

Los agentes que intervienen en la EIA son el promotor, que vela por el funcionamiento de la actuación, del que dependen el equipo de proyectistas y el equipo de ambientalistas (redactores del estudio de impacto ambiental), la autoridad sustantiva, alineada junto al promotor para el funcionamiento correcto del proyecto, el órgano ambiental, que es el valedor del entorno, y la población afectada por el proyecto.

Existe una cierta contraposición, al menos dialéctica, entre el proyecto/actuación y el entorno, que se transmite a la relación promotor/autoridad sustantiva -Órgano ambiental y que se arrastra hasta la relación proyectistas (redactores del proyecto)- ambientalistas (redactores del estudio de impacto ambiental). Tal dicotomía, particularmente en el nivel proyectistasredactores del estudio de impacto ambiental, es inaceptable. Este hecho se agrava, además, porque el proyecto, que define la obra, tiene preponderancia sobre el estudio de impacto ambiental, que la contextualiza en el territorio. Los proyectistas suelen ser ajenos a una concepción ambiental del proyecto, entendiendo el medio ambiente como una variable exógena; el centro de sus preocupaciones lo ocupa el sistema proyectado, siendo el entorno una especie de enemigo a vencer. En ocasiones se ha constatado que ciertos proyectistas manifestaban, a priori, su total indiferencia al estudio porque ya habían decidido la solución, de tal manera que sólo se aceptarían los resultados del estudio de impacto ambiental si coinciden con ella. Este estado de cosas viene determinado por la superposición de varias circunstancias:

- Carencia de la suficiente sensibilidad en relación con los problemas ambientales. 


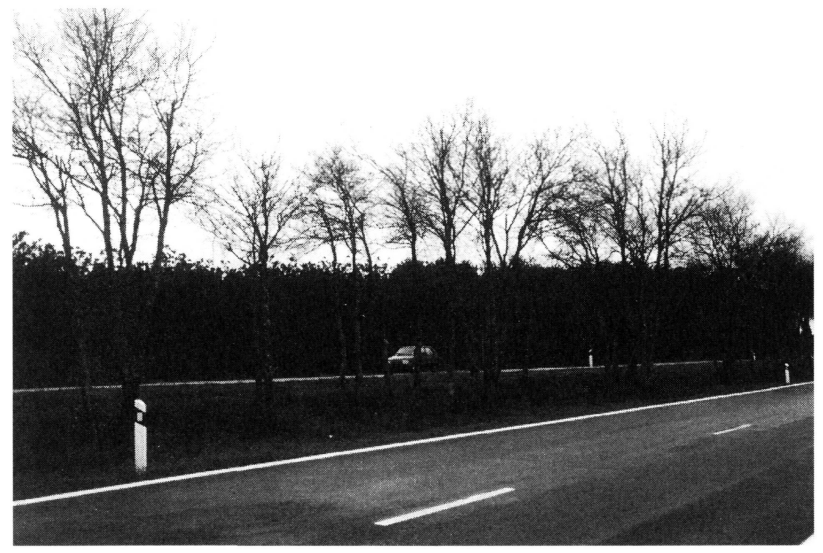

Foto 1.-Mediana en Landas francesas que conserva los robles autóctonos, en contraste con los pinos cultivados del entorno.

- Carencia de la necesaria formación en los métodos de estudio, valoración e integración ambiental y falta de datos operativos en muchos casos.

- Falta de comprensión de la unidad proyecto/entorno.

- Carencias ambientales en las instrucciones, normas técnicas, reglamentos, etc. que regulan la redacción de los proyectos.

- Traslado de la responsabilidad al equipo de ambientalistas que se ocupará del estudio de impacto ambiental.

En contra de lo que se suele pensar no es el mínimo coste, al menos no lo es siempre en el caso de las carreteras, lo que decide la alternativa/diseño a seleccionar; antes bien parecen aunarse distintos agentes que pueden, por el contrario, decidir los proyectos más caros: los proyectistas desean un proyecto "prepotente"; los constructores, contratistas de maquinaria, etc. apoyan el de mayor volumen inversor, lo mismo ocurre con la población de la zona (restaurantes, hoteles, obreros, etc.) y la Administración, en los últimos años al menos, ha contado con presupuestos generosos.

\section{LA IGNORANCIA DE LA POSIBILIDAD DE GENE- RAR IMPACTOS POSITIVOS}

Por otra parte raras veces se entiende la actuación como elemento de mejora ambiental. Y sin embargo en muchas zonas profundamente degradadas, las infraestructuras de comunicación constituyen la única posibilidad de creación de pasillos verdes capaces de proporcionar una impronta grata al paisaje. En el

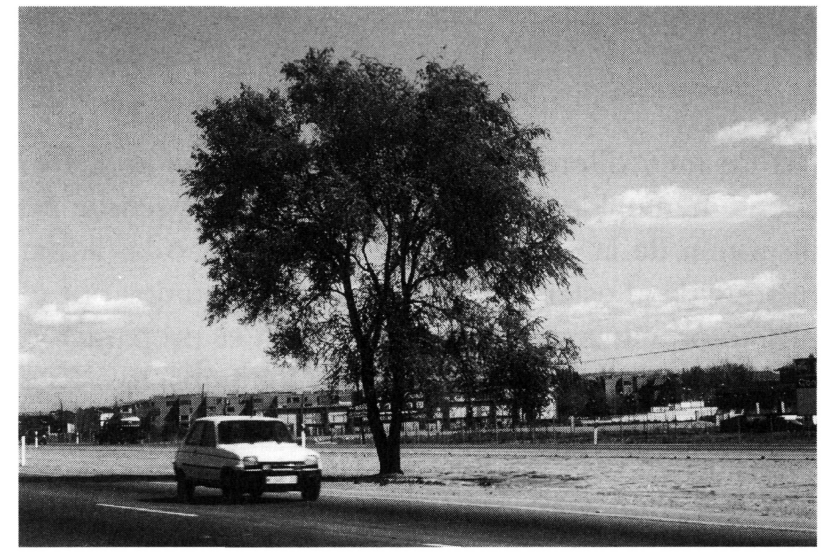

Foto 2.-Mediana en la N-II española. Sólo se conserva una acacia y se compacta toda la mediana sin otro acabado.
Reino Unido más del 50\% de la vegetación arbolada se encuentra en los terrenos de las carreteras no ocupados por las calzadas. En muchas ocasiones la obra civil en sí es un elemento realzador del entorno; recuérdese, en este sentido, el magnífico aspecto de los muros de contención con fábrica de mampostería en seco que se realizan en algunos países con programas de empleo intensivo de mano de obra (hemos tenido oportunidad de contemplar espléndidas realizaciones en la República de Cabo Verde, por ejemplo), o algunas carreteras españolas construidas en épocas pasadas. El nuevo plan de accesos a Madrid utiliza la idea de pasillo verde a lo largo de las autovías dotando a las previstas, en las áreas desforestadas del sur, de unas bandas vegetadas a ambos lados y en las medianas que definirán una malla verde; este loable planteamiento inicial puede defraudar las expectativas despertadas si razones presupuestarias impiden su materialización: los proyectos quedan muy definidos en todos los detalles constructivos excepto en éste (fotos 1 y 2).

Tampoco existe tradición y formación en relación con la búsqueda de múltiples soluciones al problema que se trata de resolver, contentándose frecuentemente el proyectista con la adopción de una solución funcionalmente correcta. En general se carece de cultura de generación explícita de un conjunto elevado de alternativas, tanto de trazado como de diseño.

\section{LA DIFICIL INDEPENDENCIA DE LOS EQUIPOS QUE SE OCUPAN DE LOS ESTUDIOS DE IMPACTO AMBIENTAL}

Los equipos responsables de la elaboración de los estudios de impacto ambiental, formados por perso- 
nas de muy diferente sensibilidad, formación y, frecuentemente, ideología que el equipo proyectista, no disponen de la necesaria independencia, o la tienen restringida al estar seleccionados y retribuidos por el promotor. Otras veces lo que les falta es preparación o criterio para valorar/interpretar los impactos. Este hecho tiene gran relevancia porque tal interpretación está impregnada de personalismo, requiriendo, de un lado, criterio, basado en una fuerte preparación y experiencia, y de otro, absoluta independencia respecto al promotor/cliente.

\section{LA INSUFICIENTE PARTICIPACIÓN PÚBLICA}

La participación pública puede considerarse escasa durante el proceso, tanto de elaboración del proyecto como del estudio de impacto ambiental, e ineficiente cuando se realiza al final de tal proceso. Valga como ejemplo una alegación presentada al estudio informativo de la Autovía del Noroeste, en su tramo Villafranca-Lugo:

“(...) Que el plano que se presenta es ininteligible para el público general, más bien lo será para ingenieros y técnicos (...) Como habla en chino, lenguaje que se ignora por los gallegos, y el proyecto es tan abstracto (...) por lo que suplica se acuerde ampliar el proyecto para hacerlo más explicativo e inteligible para "público" a que está destinado."

\section{LA AUSENCIA DE DIRECTRICES POR PARTE DEL ÓRGANO AMBIENTAL}

Frecuentemente el equipo redactor del estudio de impacto no recibe del órgano ambiental, o la recibe con retraso, la respuesta a las consultas previas que se hacen a personas e instituciones interesadas, ni directrices para la realización del estudio, incumpliendo lo que especifica el Reglamento en sus artículos 13 y 14 . Esto, que supone una dejación de funciones por parte del órgano ambiental, puede obedecer a varias causas:

- El órgano ambiental ha omitido el trámite de iniciación de consultas establecido en el artículo 13.

- Las personas o instituciones consultadas no responden; resulta curioso observar la escasez de respues- ta a estas consultas previas incluso por colectivos ecologistas.

- Los funcionarios del órgano ambiental carecen de criterio, lo que no es raro (según se ha constatado) en algunas comunidades autónomas.

- Con frecuencia las respuestas aportan, más que sugerencias o informaciones útiles, juicios de valor que pueden condicionar el estudio.

\section{LA INTERVENCIÓN DEMASIADO TARDÍA DE LA EIA}

La experiencia hasta ahora parece indicar que la EIA entra demasiado tarde en el proceso de toma de decisiones sobre el proyecto, particularmente en los proyectos de carreteras. En muchos casos el proyecto está decidido no sólo en cuanto a su ejecución (lo que ya de entrada invalida la consideración de la alternativa "cero"), sino en términos de su localización, tecnología y diseño. De esta forma el papel de la EIA queda fuertemente limitado, tan sólo puede introducir ciertas mejoras de carácter complementario y periférico, sin posibilidad de entrar en los aspectos sustantivos del proyecto.

En otras ocasiones la aportación temprana de consideraciones ambientales a la redacción del proyecto cae en la inoperancia porque el órgano ambiental es incapaz de exigir y controlar su incorporación en fases posteriores.

Por último es de destacar el hecho de que alguna legislación autonómica de carreteras (por ejemplo Canarias) sitúa expresamente la realización del estudio de impacto ambiental sobre el proyecto de construcción (foto 3).

\section{DESVINCULACIÓN DEL TERRITORIO}

Los impactos ambientales están estrechamente ligados a la localización, más en el caso de las carreteras; prácticamente todos los impactos de estas infraestructuras pueden resolverse a travéés de la ubicación de la traza, por lo que resulta absolutamente indispensable la intervención de la EIA en la búsqueda de pasillos para los ejes viarios. 


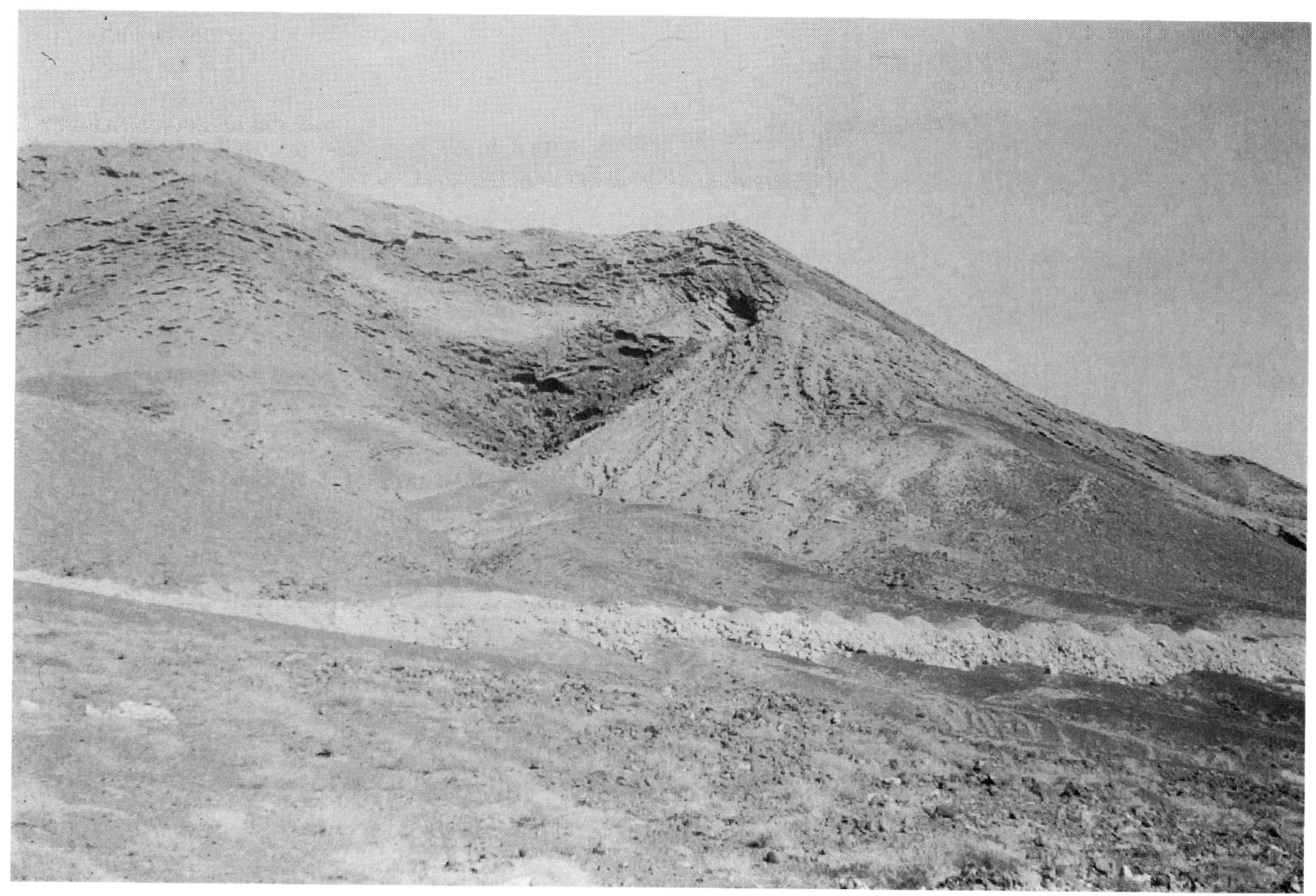

Foto 3.- En Canarias, a pesar de la Legislación, se abre una carretera al pie mismo de una caldera volcánica.

\section{LA CONFUSA REGLAMENTACIÓN DEL PROGRAMA DE VIGILANCIA AMBIENTAL}

Un aspecto insuficientemente desarrollado/concretado en el Reglamento de EIA es el programa de vigilancia ambiental y, consiguientemente, mal resuelto en los estudios realizados. Tampoco queda suficientemente precisado el nivel de detalle a que deben definirse las medidas protectoras, correctoras y compensatorias, en su caso, ni el tipo de documento en que deben recogerse: proyecto sustantivo, proyecto independiente, pliego de condiciones, etc. Asimismo habría que regular la forma de control sobre la aplicación real de tales medidas.

En ocasiones estas medidas se aplican a destiempo, resultando costosas e ineficaces; tal es el caso de la variante de Aranjuez donde se ejecutaron las obras de restauración ambiental dos años después de inaugurada la carretera, cuando ya se habían producido daños de difícil reversibilidad.

\section{SOBRE LOS FACTORES}

La Directiva enumera los factores ambientales e incluye unos aspectos, los "bienes materiales" de sig- nificado difuso. La enumeración de factores ambientales que hace el Reglamento difiere algo de la anterior. En todo caso hay una cierta confusión en relación con los factores a considerar. En concreto los de tipo socioeconómico parece que sólo deberían ser considerados en la medida en que impliquen repercusiones ambientales claras: por ejemplo la generación de empleo y consiguiente fijación de población en zonas rurales deprimidas. Pero este factor no debería ser considerado en el caso de las carreteras, siendo más propio de la evaluación socioeconómica de éstas.

\section{SOBRE LOS EQUIPOS}

En el país existe un claro déficit de personal preparado para realizar, con criterio y autoridad, los estudios de impacto ambiental. Por otra parte, no siendo el tema específico de ningún colectivo profesional, el campo se ha llenado de diletantes y arribistas, dispuestos a realizar cualquier estudio por cualquier precio. Hay que considerar que los estudios de impacto ambiental suelen ser caros, pofque requieren mucho trabajo de campo, muches datos y la intervención de numerosos profesionales de alto nivel. Sin embargo no están adecuadamente pagados. 


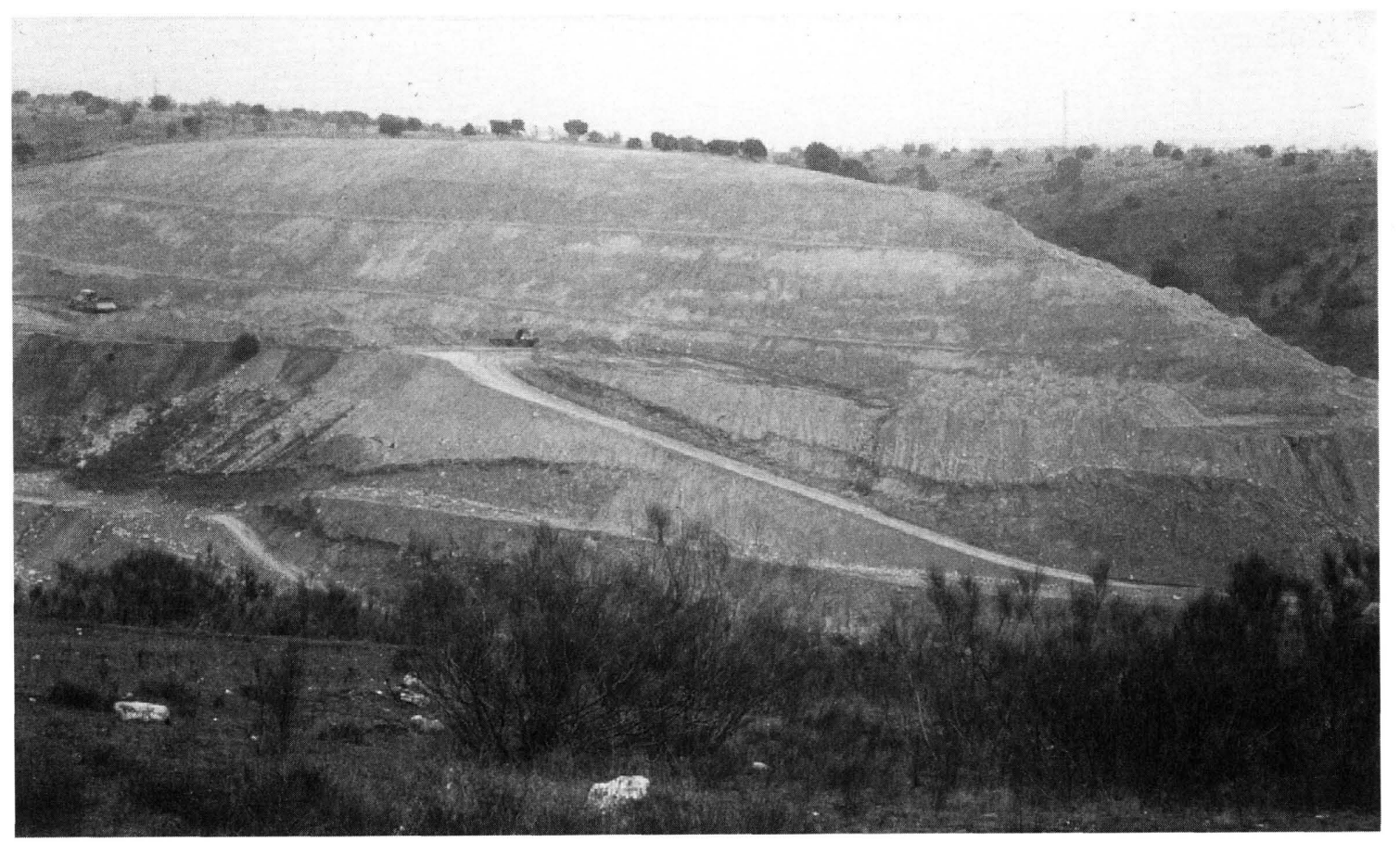

Foto 4.- Terrible desbronce y consiguiente movimiento de tierras.

Por otra parte no existe, en general, un adecuado control de calidad, siendo en la práctica indiferente hacer un estudio malo o bueno. A título de anécdota, el autor de un estudio ha utilizado el antiquísimo método de Batelle tan al pie de la letra, que en la casilla donde pone "indios" contesta "no hay".

Todo lo anterior no favorece, antes al contrario, que la realización de los estudios se encomiende a las personas y equipos más capaces.

\section{SOBRE LOS DATOS}

Existe carencia de datos básicos de carácter descriptivo, pero sobre todo valorativo. A ello se añade el hecho de que muchos de los existentes no están publicados y la actitud reacia a proporcionarlos por parte de quienes disponen de ellos: departamentos universitarios, centros de investigación y otros organismos públicos; no hace falta señalar la actitud de los privados.

\section{CARENCIA DE SENSIBILIDAD Y FORMACIÓN AMBIENTAL POR PARTE DE LOS RESPONSABLES DEL PROYECTO}

Se tiende a confundir el medio ambiente con "los pajarillos y las florecillas" sin entender la poderosa filosofía que subyace en el hecho ambiental. La exigencia de la consideración ambiental debería aparecer en las instrucciones para la realización de proyectos y obras, en pie de igualdad, por ejemplo, con las instrucciones sobre el hormigón. Incluso cargos de la más alta responsabilidad atribuyen el mayor riesgo de accidentes, en cierta curva de la variante de Aranjuez, a la necesidad de ejecutar la obra de tal manera que se protegieran ciertas "mariposillas", cuando las restricciones de diseño necesarias para su protección distan unos 5 kilómetros del punto negro señalado y en ningún momento obligaron a forzar el trazado.

Un proyecto que levantaba millones de t de tierra de túneles y taludes como era la variante de NavarraPamplona a lo largo del valle del Araxes no incluía lugares dónde depositar estos excedentes. Interpelado el proyectista que dónde se haría el vertido contestó: "En cualquier sitio".

\section{METODOLOGÍA}

A pesar de que el propio Reglamento propugna la cuantificación de los impactos, la tendencia se orienta a las valoraciones cualitativas sin explicitar crite- 
rios en que se apoyen los juicios de valor. Por otro lado se encubre la pobreza de datos relevantes y de criterios claros con la aportación de descripciones inútiles de plantas, listas de animales y otros muchos datos de la más absoluta inoperancia.

\section{DISCRIMINACIÓN DE LOS PROMOTORES PRIVADOS FRENTE A LOS PÚBLICOS}

Resulta inaceptable que la Administración sea más exigente con los proyectos de promoción privada que con los de promoción pública. En este sentido resulta chocante el escaso cumplimiento de los plazos establecidos por parte de los respectivos órganos ambientales; conocemos casos en que la dilación que ello supone, por un exceso de celo o de desconfianza, desanima a inversores con proyectos favorables.

\section{EL SUPUESTO POTENCIAL DE DESARROLLO ESPAÑOL}

A España se le ha reconocido un alto potencial de desarrollo por su situación, capital humano, territorial, científico, cultural, etc., si bien con un fuerte déficit de infraestructuras que estrangulan su materialización. Por otro lado las infraestructuras de transporte son el principal elemento vertebrador y articulador de la política territorial, hasta el punto de que las áreas alejadas de un eje de desarrollo están condenadas a la depresión. Por todo ello en los últimos años ha habido una importantísima inversión en infraestructuras de transporte, operando sobre una consigna que, aunque no explicitada, ha sido muy real: el control ambiental no puede suponer una rémora para la rápida ejecución de tales infraestructuras. En consecuencia la aplicación de la EIA no puede considerarse satisfactoria; así nos encontramos con:

- Prioridad/urgencia de la actuación frente a cualquier otra consideración.

- Prepotencia del organismo responsable de las carreteras y de los técnicos encargados de la redacción del proyecto.

- Carácter reactivo, y por tanto antipático, de la EIA tal como está legalmente regulada. Los proyectistas se resisten a cambiar el proyecto y, en todo caso, las modificaciones son caras y difíciles.
- Vinculación del estudio de impacto ambiental al órgano sustantivo.

- Incorporación tardía de las consideraciones ambientales; incluso el Reglamento de EIA sitúa la intervención de ésta sobre el documento en el que se decide la ubicación de la traza, reduciendo, así, la posibilidad de intervenir en la búsqueda de soluciones al trazado. En algunas comunidades autónomas (por ejemplo, Canarias) se aplica incluso al proyecto de construcción.

\section{DESCOORDINACIÓN ADMINISTRATIVA}

La dependencia de un mismo Ministerio, en el nivel nacional, del órgano ambiental y de la autoridad sustantiva, no parece propiciar la ruptura de la dualidad proyecto-entorno y la consiguiente colaboración, antes bien parece existir, o haber existido al menos, una animosidad entre ambas Direcciones Generales (carreteras y medio ambiente), una especie de celos competenciales que se ha manifestado, por ejemplo, en el veto de la D.G. de Medio Ambiente a aportaciones técnicas y publicaciones de la D.G. de Carreteras sobre metodologia de EIA.

En contrapartida las exigencias del FEDER, que ha financiado muchas de estas infraestructuras, han supuesto un correctivo al estado de la cuestión. Resulta paradigmático el caso de la autovía AlmeríaMurcia que atravesaba el famoso Karst Yesífero de Sorbas, lo que creó numerosos problemas que podrían haberse obviado con un mero inventario de los puntos más sensibles o con la incorporación de un ambientalista a la fase de generación de alternativas de trazado.

\section{ALGUNOS CAMBIOS ESPERANZADORES}

El estado de cosas parece estar cambiando en la actualidad, al menos en algunos casos; así el plan de accesos a Madrid, que comprende una fortísima inversión en nuevos cinturones y desdoblamiento de los accesos radiales, ha incorporado desde el primer momento el equipo de ambientalistas al equipo de proyectistas trabajando codo a codo en la búsqueda de las trazas en cada uno de los tramos. Se partió, en un primer momento, del señalamiento de puntos/zonas sensibles, luego se realizé un inventario y valoración del territorio y sobre esta información se generaron y evaluaron, en ciclos continuos y 
rápidos, multitud de alternativas que al final quedaron reducidas a una sola en muchos casos y a unas pocas en los puntos más conflictivos, las cuales se someten después a un proceso más detallado de evaluación. De esta forma la mayor parte de los problemas ambientales han quedado obviados en esta fase previa, limitándose después la EIA a la profundización en ciertos aspectos y a una especie de refino del proyecto.

No obstante lo correcto del planteamiento metodológico, la experiencia no puede considerarse del todo positiva, por la eterna cuestión de que el órgano sustantivo y los propios proyectistas siguen considerando al medio ambiente como un tema menor, que sólo se considera en la medida en que está de acuerdo con los otros criterios de diseño.

También en el caso de Madrid se está considerando la intervención de los numerosos ejes viarios que discurren por el sur como elementos de mejora ambiental mediante la incorporación de bandas verdes a ambos lados y en la mediana de los ejes viarios. Por último hay que llamar la atención sobre la falta de perspectiva que puede generar sobre el impacto total la división de una carretera en tramos para su evaluación ambiental. A ello puede inducir la ignorancia de impactos acumulativos o sinérgicos derivados del proyecto global.

\section{LA INTEGRACIÓN AMBIENTAL DE LOS PROYECTOS: GESTIÓN ADAPTATIVA DE LA EIA}

Generación de nuevos proyectos. En general la idea de un proyecto surge para resolver un problema: satisfacer una aspiración, aprovechar una oportunidad o poner en valor un recurso ocioso. El proyecto puede derivarse de la presión social sobre la iniciativa pública o del interés que se presenta a la iniciativa privada.

En general una actuación pasa por varias fases: Estudios Previos, incluyendo proyecto (en el caso de carreteras, según el Proyecto de Reglamento, éstos pueden ser Estudio de planeamiento, Estudio previo, Estudio informativo, Anteproyecto y Proyectos de construcción y de trazado), fase de construcción, fase de explotación o funcionamiento y, en ciertos casos, fase de abandono. Todas ellas han de estar imbuidas de la temática ambiental.

La actuación puede estar contemplada en un plan previo, coordinada con otras actuaciones o surgir de forma aislada. El proyecto del Reglamento de Carreteras prevé un Plan de Carreteras: es en éste donde la consideración del hecho ambiental producirá mejores resultados; ya se indicó, por otro lado, la postura favorable del V Programa de Acción a la vinculación de los planes a EIA. Si el Plan ha estudiado la capacidad de acogida del medio, y decidido en consecuencia, los proyectos que de él surjan estarán ambientalmente integrados. Cuando la actuación no esté prevista en un plan, su concepción deberá estar orientada por un estudio ambiental previo del entorno en que se ubica.

\section{INCLUSIÓN TEMPRANA DE CONSIDERACIONES AMBIENTALES}

La Directiva 85/337 CEE, en coincidencia con la opinión de técnicos y científicos, subraya que la mejor política de medio ambiente consiste en evitar, desde el principio, la creación de contaminaciones o daños, más que combatir posteriormente sus efectos y la necesidad de tener en cuenta, lo antes posible, las repercusiones sobre el medio ambiente en todos los procesos técnicos de planificación y decisión.

Ello dentro de un proceso de integración ambiental que ha de producirse en todos los hitos del proceso general de toma de decisiones: en la formulación de políticas, en las disposiciones legales y administrativas, en los planes, en los proyectos, en la ejecución y en la gestión.

La ubicación de la EIA en el proceso de toma de decisiones determina tres enfoques:

Reactivo, cuando se sitúa la EIA tras la decisión de ejecutar el proyecto; es una práctica no recomendable porque de los tres tipos de decisión a que, bien utilizada, puede conducir la EIA: aceptación, modificación o rechazo. En este caso sólo es real la aceptación (difícil) y, en tal caso, ineficaz la modificación e impensable el rechazo del proyecto. El proceso de toma de decisiones en este supuesto pasa por las siguientes fases:

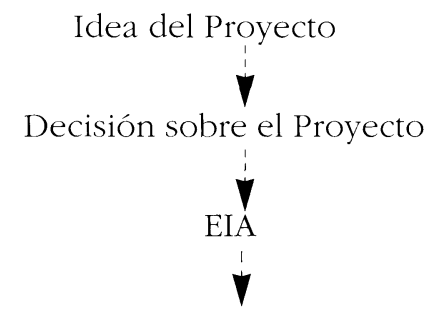

Decisión $\left\{\begin{array}{l}\text {-Generalmente acep̄tación } \\ \text {-Difícil modificación } \\ \text {-Rechazo impensable. }\end{array}\right.$ 
Semiadaptativo, cuando la EIA se sitúa antes de decidir la ejecución del proyecto, tal como indica el esquema adjunto. Este enfoque es el que se viene practicando en la actualidad y es el que implícitamente supone la aplicación de la legislación vigente.

Idea del Proyecto

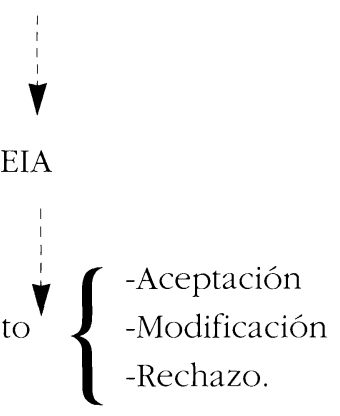

Adaptativo, este enfoque considera que todo proyecto debe estar contemplado en un plan previo, en su normativa o en su programa de actuaciones y, por tanto, o bien se encuentra ambientalmente integrado o el plan especificará, en su caso, el alcance y contenido de la EIA, orientándola hacia los aspectos más importantes o conflictivos. Es el enfoque recomendado por los teóricos del medio ambiente. En este caso, las fases del proceso de toma de decisiones serían:

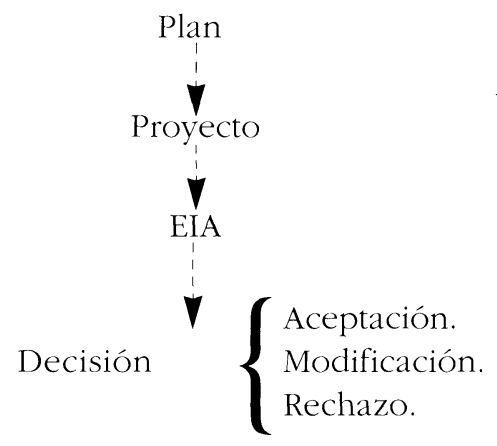

\section{ESQUEMA DE INTEGRACIÓN}

La integración ambiental podría realizarse según los siguientes pasos genéricos:

1). Estudio del medio/inventario ambiental, a escala pequeña, dependiendo de la entidad del proyecto, entre 1:25.000 y 1:100.000. En términos estimativos el inventario contendría la toma de datos básicos, la definición y delimitación de ecosistemas o de unidades ambientales sobre el territorio, una valoración de los méritos de conservación, un entendimiento de las interacciones entre elementos y entre zonas, una estimación de la fragilidad y potencialidad del medio y por fin una estimación de la capacidad de acogida de cada punto o unidad para la actuación de que se trate. Con carácter previo podría incluirse la localización de puntos más sensibles/valiosos que deben ser evitados a escala más pequeña (1:100.000 ó 1:200.000). Esta primera tarea se obviará cuando el proyecto esté contemplado en un plan previo.

2). Concepción del proyecto de acuerdo con el análisis anterior, afectando al carácter de la localización de la traza o pasillo, de los préstamos y caballeros, de las obras auxiliares, etc., al diseño en términos de perfil transversal fundamentalmente (bandas verdes, vermas, etc.) de las obras de fábrica, muros de contención, etc. considerando el posible efecto positivo del proyecto en el paisaje, a la tecnología a utilizar (maquinaria y equipos, mano de obra, excavaciones, explosiones, etc.). Estas consideraciones justifican la importancia de intervenir en la sensibilización y formación de los proyectistas, así como de hacer intervenir criterios ambientales en la legislación sectorial que define el carácter de las actuaciones en cada sector (foto 5 ).

Esta última aseveración no es sino la aplicación del Nuevo Tratado de Unión Europea cuando señala (letra k, art. 3) que las exigencias de protección ambiental deben integrarse en la definición y realización de las demás políticas. Por otra parte el Libro Verde del impacto del Transporte fía la adecuación ambiental del transporte a la adopcion de medidas dentro del sector y de acuerdo con el concepto de movilidad sostenible.

3). Generación de múltiples alternativas, principalmente de localización, pero no exclusivamente de evaluación/selección posterior. La EIA se fundamenta más que en otra cosa en la evaluación de alternativas, incluyendo la alternativa "cero"; paso previo a tal evaluación será la generacion de alternativas, si bien ambas tareas no han de entenderse como fases secuenciales sino que interactúan mutuamente sucediéndose en forma cíclica. Esta tarea ha de realizarse a la misma escala que el inventario ambiental. Al final del proceso de generación/selección quedarán unas pocas alternativas, particularmente afectando a lugares o tramos más conflictivos, que habrán de someterse a un análisis sistemático de evaluación. Existen modelos de generación de alternativas, algunos sistemáticos (modelo Impacto-Aptitud, Superposición de transparencias, modelo factorial, etc.) y otros empíricos; también existen modelos de evaluación de alternativas con la característica 


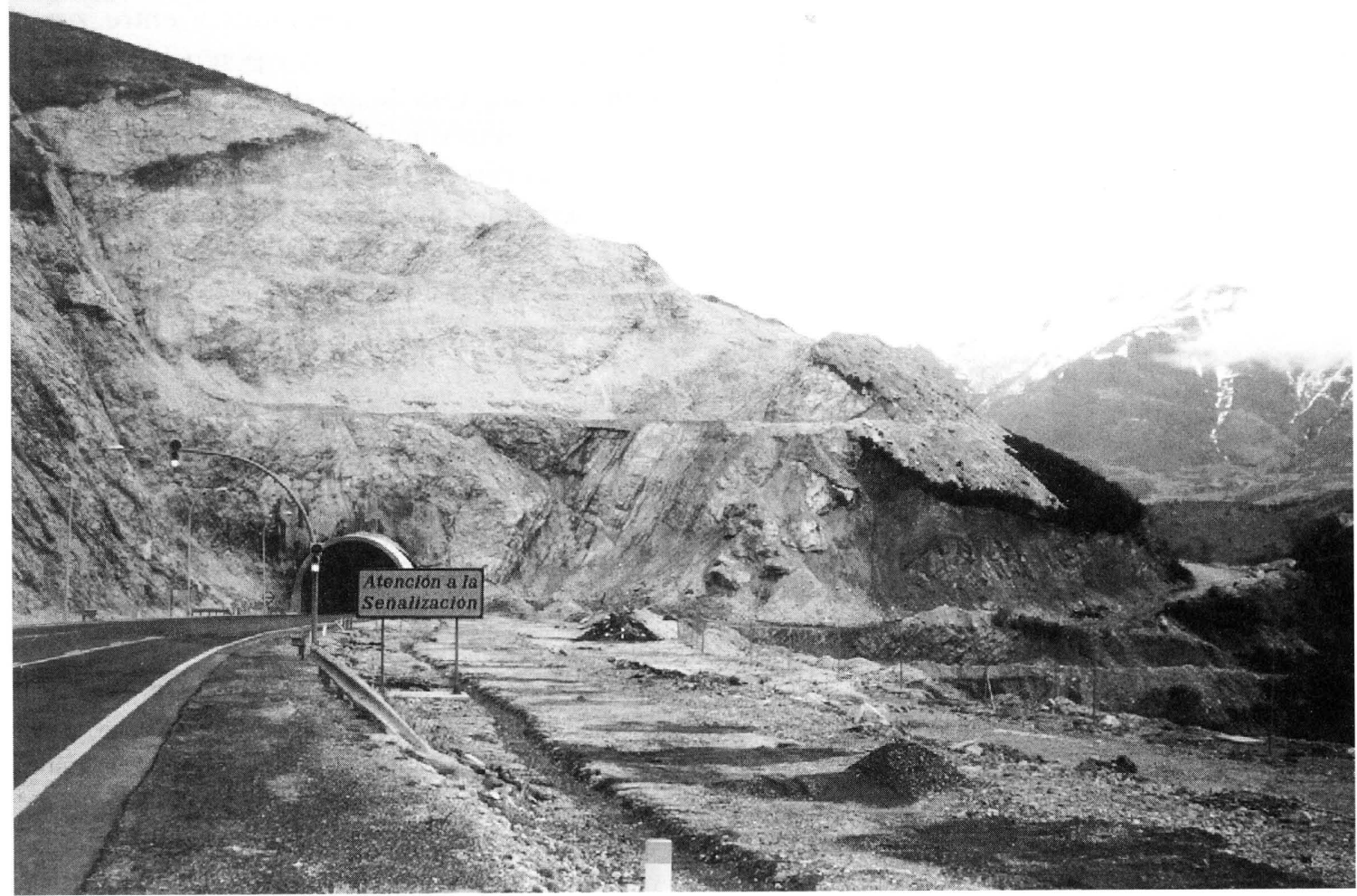

Foto 5.- Zona frágil desmontada excesivamente sin aparente necesidad para un sencillo túnel.

común de que han de ser multicriterio. Ambos casos exigen equipos multi e interdisciplinares con intervención de los clásicos proyectistas y del equipo de ambientalistas responsables de la evaluación del impacto ambiental.

En este momento podría estar recomendado realizar una primera declaración de impacto ambiental.

4). Redacción de anteproyectos y/o proyectos técnicos sobre la alternativa seleccionada, teniendo en cuenta todos los elementos y consideraciones ambientales deducidos de las tareas precedentes. Habría de nuevo participación de ambientalistas poniendo el énfasis en la calidad integral del proyecto; debe tenerse en cuenta que el medio ambiente puede ser un poderoso elemento de innovación tecnológica respecto al proyecto.

5). Evaluación detallada de impacto ambiental. Esta tarea quedaría reducida a la profundización en aspectos muy concretos, a la elaboración del programa de vigilancia ambiental y del documento de síntesis final, en la idea de que la mayor parte de los impactos habrán sido prevenidos/corregidos/compensados a lo largo del proceso de integración descrito.

(c) Consejo Superior de Investigaciones Científicas Licencia Creative Commons 3.0 España (by-nc)

\section{INTERNALIZAR LOS COSTES AMBIENTALES}

El proyecto ha de internalizar los costes ambientales, aquellos en los que incurre por las degradaciones que produzca en los elementos y procesos naturales y aquellos que se introduzcan por la adopción de las medidas correctoras o compensatorias. El V Programa de Acción señala a este respecto la inclusión de los costes reales (que incluye los ambientales) en las políticas y decisiones de inversión, así como en los costes que recaen sobre el usuario.

En el caso de que el impacto sea aceptable debe responderse a la cuestión de si dispone de las mejores medidas correctoras; la respuesta afirmativa conduce a la aceptación del proyecto, mientras que la respuesta negativa requeriría la introducción de tales medidas correctoras para dicha aceptación. De acuerdo con el Libro Verde sobre el impacto del transporte la reducción de la denominada "contaminación operativa", exige la aplicación de la "mejor tecnología disponible" a los modos de transporte, a la calidad del carburante y a las infraestructuras.

El impacto inaceptable no conduce directamente al rechazo del proyecto, sino que antes debe cuestionarse si existen razones socioeconómicas que pue- 


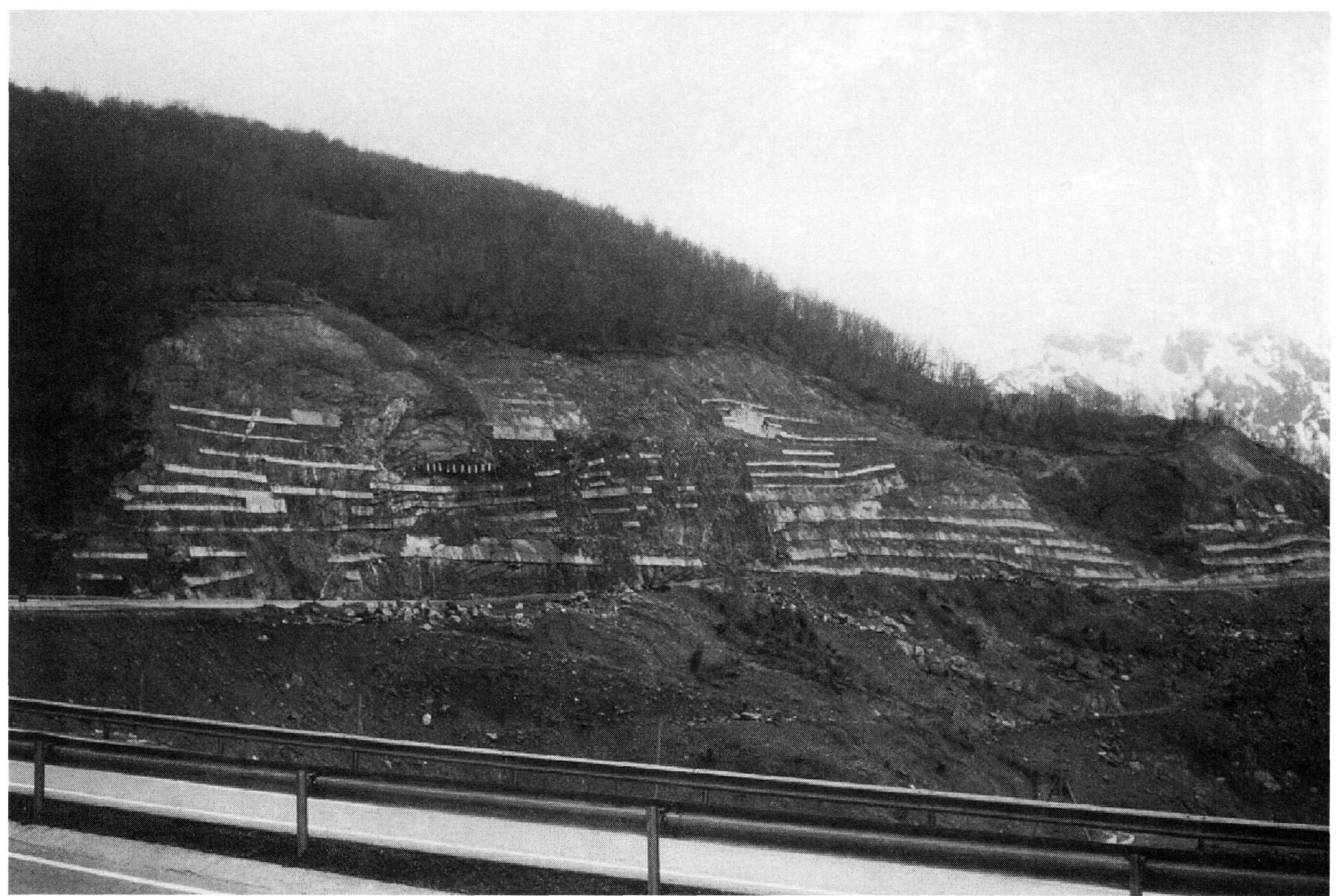

Foto 6.- La falta de respeto al medio crea, a veces, un paisaje tan lamentable como éste. Autovia de Asturias.

dan justificarlo, pudiendo aceptarse el proyecto en caso afirmativo.

\section{LA INTEGRACIÓN AMBIENTAL DE LAS CARRETERAS}

Las fases expuestas deben entenderse como genéricas para cualquier tipo de proyectos. En el caso de carreteras el Proyecto de Reglamento específica una serie de fases desde el plan hasta los proyectos de construcción y trazado. La integración ambiental podría acompasarse a cada una de tales fases, tal como se esboza a continuación.

- Plan de Carreteras del Estado. Aquí la integración ambiental se haría básicamente a partir de la definición y delimitación a pequeña escala de las zonas de mayor valor y fragilidad, así como de aquellas otras donde las carreteras podrían ser un elemento creador de paisaje.

- Estudio de planeamiento. Aquí debe completarse el inventario de los puntos más valiosos y frágiles con un inventario y valoración general, bien de todo el territorio, bien de unos pasillos amplios a lo largo de los ejes previstos en el esquema vial. Se sugiere que se incluya este tipo de información como conte- nido normativo de los estudios de planeamiento en el Reglamento de Carreteras.

- Estudio previo. Éste tiene como finalidad, según el proyecto de Reglamento de Carreteras, "la recopilación y análisis de los datos necesarios para definir en líneas generales las diferentes soluciones a un determinado problema, valorando todos sus efectos". Entre los datos a recoger no mencionan los ambientales, cuando su inclusión parece obvia. En esta fase debería incorporarse algún experto en materia de medio ambiente que incorporara el estilo ambiental a la definición/generación de soluciones al problema de que se trate y que incorporase, también, variables ambientales al proceso de selección/evaluación que establece el proyecto de Reglamento.

Estudio informativo. Tiene como finalidad definir en líneas generales el trazado de la carretera. Estamos, al parecer, en la fase de generación y evaluación de alternativas de trazado, siendo válido, por tanto, lo señalado con carácter general en el punto 19. El proyecto de Reglamento incluye como contenido normativo "la evaluación del impacto ambiental de las diferentes opciones"; habría que añadir la incorporación de las consideraciones ambientales al propio proceso de generación y evaluación, tal 
16

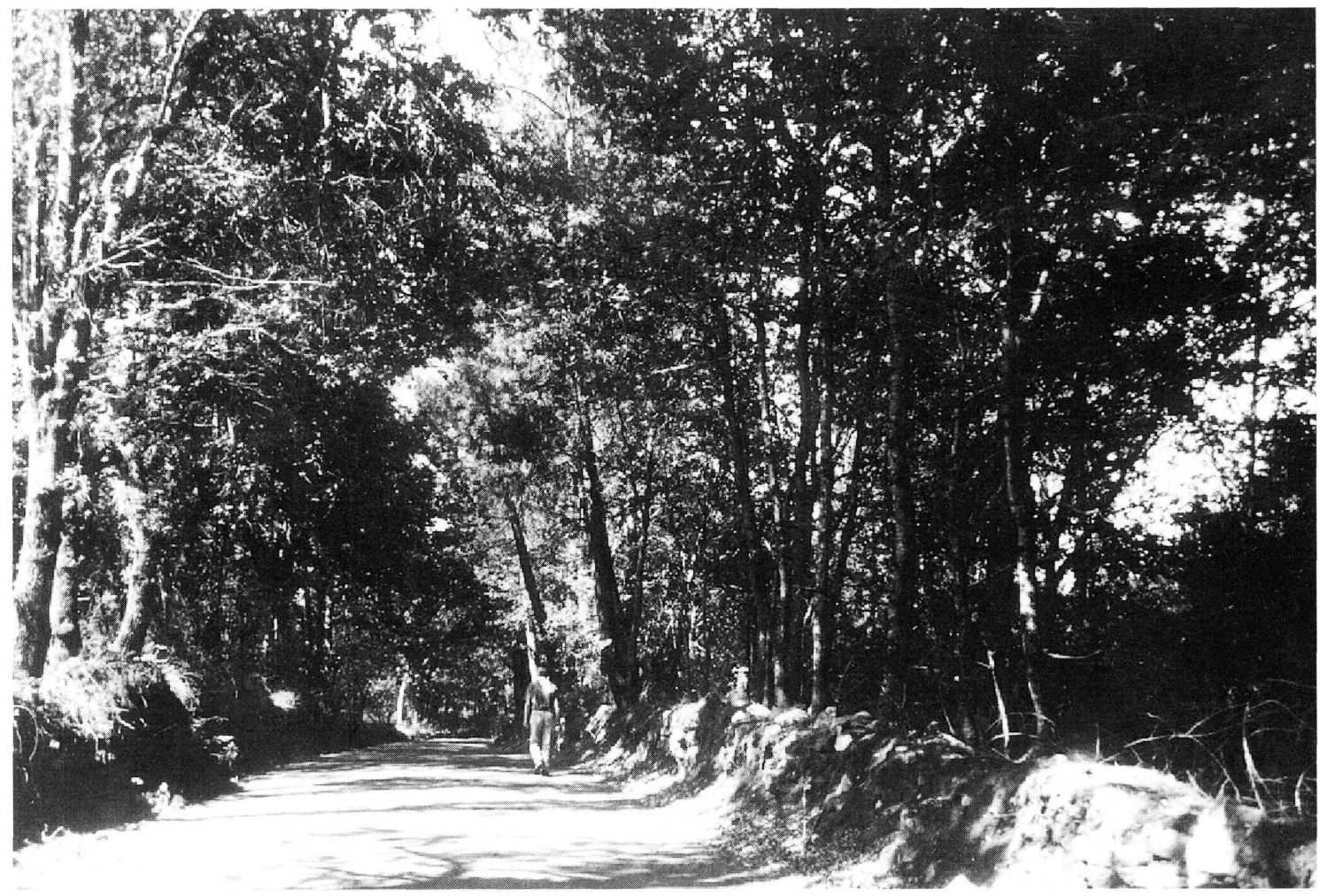

Foto 7.- Nostalgia por estas carreteras que un ensanche transformará.

como se dijo. El proyecto de Reglamento deja para la fase de proyecto el estudio de yacimientos y procedencia de materiales: parece que alguna idea sobre este tema debería darse en el estudio informativo extendiéndola, además, a los lugares de vertederos y de localización de instalaciones u obras auxiliares. También parece conveniente analizar la posibilidad de emitir sobre este estudio una declaración de impacto ambiental.

- Anteproyecto. Consiste en la concreción de la solución óptima a escala del orden de 1:5.000. De nuevo aquí la integración ambiental estriba en la concepción correcta del anteproyecto. Se fijarán aquellas medidas de corrección o compensación ambiental que deben dar objeto a proyectos parciales específicos

-Proyecto de construcción y de trazado. Concreción de la solución óptima hasta hacerla realizable. Incorporación al proyecto de todas las medidas ambientales que han ido surgiendo en todo el proceso precedente como partidas presupuestarias o en el pliego de condiciones; asimismo realización de los proyectos específicos señalados en el anteproyecto. Ciertos impactos de la carretera sólo pueden tratarse afectando a terrenos externos a su traza; en estos casos el proyecto de trazado, en el que se definen los terrenos a ocupar por la carretera y sus elementos funcionales, deberá incluir tales terrenos necesarios para la corrección o compensación de los impactos de la carretera (fotos 7 y 8 ).

\section{MEDIOS INSTRUMENTALES}

El Talón de Aquiles de la EIA es la información, tanto básica como en distintos grados de elaboración, por lo que es necesario avanzar en la recogida de información por parte de los poderes públicos y en la disposición fácil de ella al alcance de los interesados, haciendo uso de la Directiva comunitaria sobre acceso a la información.

El V Programa de Acción, en la definición de su estrategia hacia el desarrollo sostenible, amplía la gama de instrumentos incluyendo los de mercado y una mejor información, educación y formación. Respecto a la información señala como principales lagunas:

Las diferencias de nomenclatura, criterios y metodología en la recogida y análisis de datos que difi- 


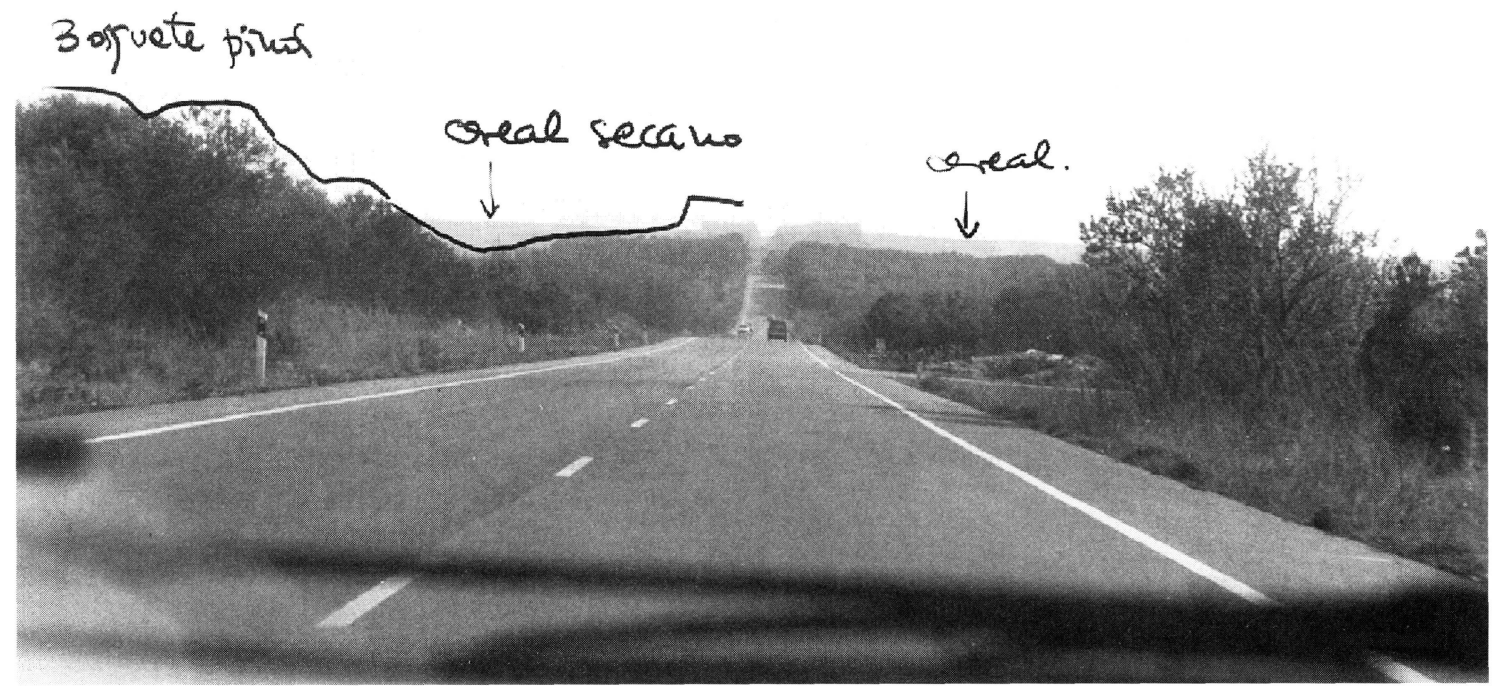

Foto 8.- En ocasiones, la carretera atraviesa la linica mancha de vegetación arbolada.

cultan la compatibilidad y comparanza en los niveles regional, nacional y comunitario.

- Grave falta de datos básicos, indicadores y demás material cuantitativo y cualitativo necesario para evaluar la situación y tendencias ambientales, para determinar y adaptar las medidas políticas y para dirigir las inversiones de capital.

- Carencia de datos cuantitativos precisos sobre las influencias del hombre sobre el medio ambiente.

- Inadecuada presentación y elaboración de la información para una fácil utilización por los destinatarios últimos.

Por ello propone colmar las lagunas en los datos básicos, aumentar su comparanza, compatibilidad y transparencia, normalizar la recogida, el cotejo y la interpretación, explotar e incrementar las experiencias y capacidades del sistema estadístico en materia de medio ambiente hasta hacerlo comparable a las estadísticas oficiales en el terreno económico-social y aumentar la frecuencia y calidad de los informes ambientales en todos los niveles administrativos.

\section{LA NECESIDAD DE SENSIBILIZAR A LOS AGENTES}

El segundo problema grave está en la sensibilización y formación de personal en los siguientes papeles: consultor/realizador de los estudios de impacto ambiental, proyectistas y funcionarios del órgano ambiental que han de juzgar los estudios presentados. Esto es más urgente en el nivel autonómico que en el estatal.

La creación de paneles de expertos independientes es otro punto de gran interés para la emisión de las declaraciones de impacto ambiental, si bien habrá que atender con cuidado a la selección de los panelistas.

\section{UNIFICACIÓN DE METODOLOGÍAS Y CRITERIOS DE VALORACIÓN, ASÍ COMO PUESTA A PUNTO DE UN SISTEMA DE INDICADORES AMBIENTALES HOMOGÉNEO}

En el caso de los proyectos públicos (y particularmente los de carreteras, de acuerdo con el Libro Verde sobre el impacto ambiental del transporte), convendría poner a punto una metodología basada 
en la relación coste/beneficio en que se incluyeran los costes y beneficios ambientales.

\section{INSTRUMENTAR LA PARTICIPACIÓN PÚBLICA}

Considerando al ciudadano como:

- Persona interesada por el medio ambiente con capacidad para influir en la toma de decisiones.

- Como generador individual de degradaciones y contaminaciones.

- Como consumidor de bienes y usuario de servicios con capacidad para elegir el producto o tipo de servicio ambientalmente más integrado, informándole adecuadamente.

\section{FOMENTO DE LA INVESTIGACIÓN CIENTÍFICA Y EL DESARROLLO TECNOLÓGICO}

En particular, en el transporte debe favorecerse la investigación y el desarrollo destinados a mejorar el resultado ambiental de los diferentes modos de infraestructura de transporte y nuevas tecnologías energéticas (biomasa, energía solar, vehículo eléctrico, etc.). También se precisa investigación orientada a la expresión económica del valor de los recursos naturales y de las degradaciones.

\section{MEJORA DE LA PLANIFICACIÓN TERRITORIAL}

Y SECTORIAL

Es ésta una asignatura pendiente en España que requiere sensibilidad pública y preparación técnica.

\section{publicaciones del ICCET/CSIC}

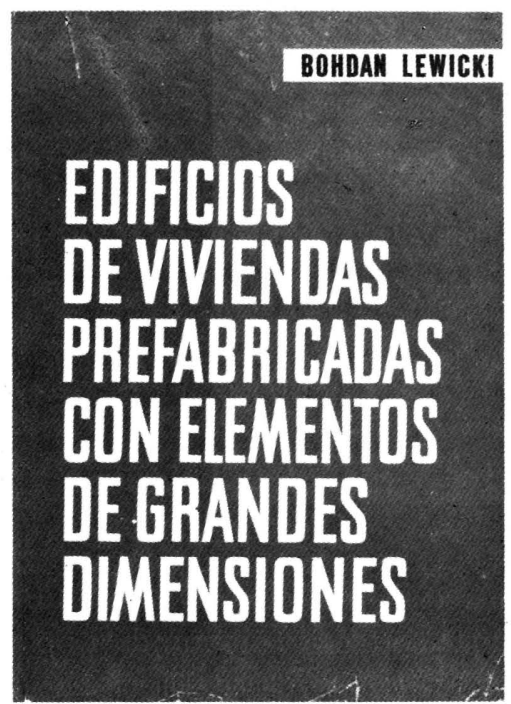

\section{Bohdan Lewicki}

Este libro trata de los problemas relativos a la construcción de los edificios de viviendas o publicos realizados con elementos prefabricados de grandes dimensiones. Se han estudiado los problemas de arriostramiento, así como los que plantea la resistencia de los elementos y de la estructura; se han examinado las cuestiones de orden higrotérmico, acústico y de resistencia al fuego; también se ha profundizado en el estudio de la estanquidad de los muros exteriores y de las juntas.

La obra incluye numerosas ilustraciones que dan detalles de diversas soluciones, asi como ejemplos de cálculo, tablas de valores numéricos, diagramas y ábacos.

Un volumen encuadernado en tela, de $24 \times 17 \mathrm{~cm}$, compuesto de 616 págs.

(c) Consejo Superior de Investigaciones Científicas Licencia Creative Commons 3.0 España (by-nc)
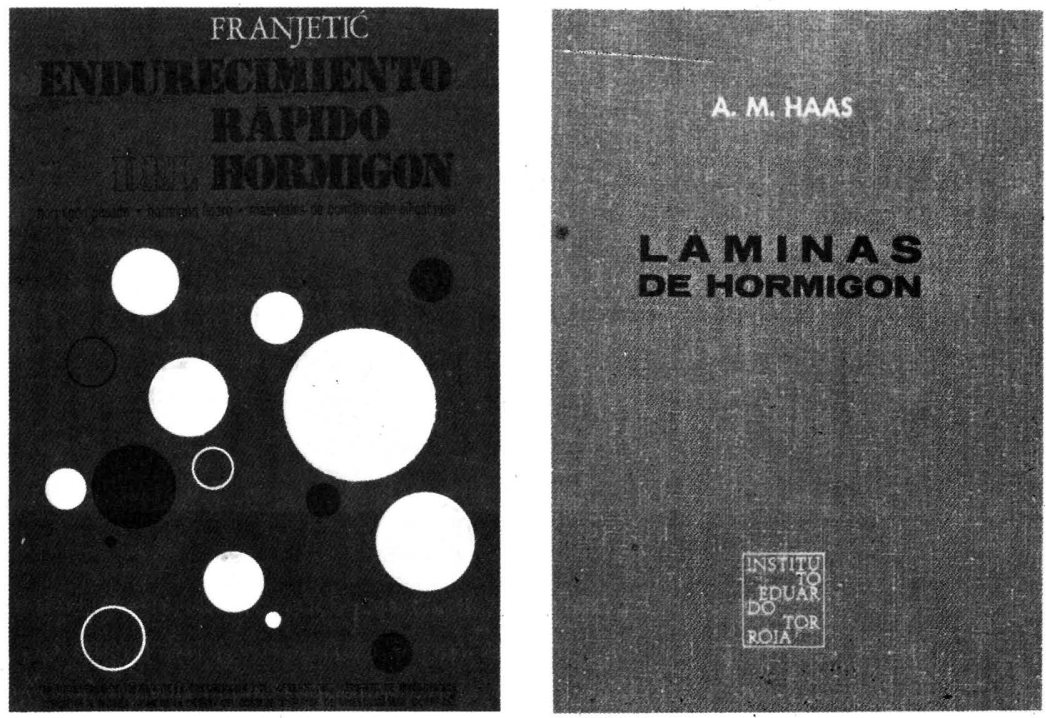

Zorislav Franjetić

En la obra de Franjetić se expone de una forma minuciosa, ordenada y sistemática, todo un cuerpo de doctrina que reúne el conocimiento actual sobre el endurecimiento nocimiento actual sobre el endurecimiento
rápido del hormigón. Parte el autor de los principios básicos y llega a las últimas consecuencias y realidades técnicas y econó micas.

Es una obra de consulta, tanto para el investigador sobre la materia, como para el proyectista y el realizador y montador de plantas e instalaciones y equipos de curado y endurecimiento rápido.

Un volumen encuadernado en cartóné, de $17 \times 24,5 \mathrm{~cm}$, compuesto de 385 págs. 110 figuras y 10 tablas.

\section{A. M. Haas}

Al escribir este libro el autor intento poner a disposición de los estudiantes y de los ingenieros unos conocimientos prácticos y construcción de láminas delgadas de hormigón.

El autor está convencido de que el éxito en el diseño de una lámina exige, por parte del
proyectista, un examen de las tres fases por las que pasa la materialización de la lámina: el diseño, el análisis estructural y la construcción de la estructura.

Un volumen encuadernado en tela, de $17 \times 24,5 \mathrm{~cm}$, compuesto de 420 págs., 141 figuras, 22 fotografias $\bar{y} 6$ tablas. adecuados para servir de guia en el diseño 